\title{
Estimation of Extinction Probabilities of Five German Cattle Breeds by Population Viability Analysis
}

\author{
J. Bennewitz ${ }^{1}$ and T. H. E. Meuwissen ${ }^{2}$ \\ ${ }^{1}$ Institute of Animal Breeding and Husbandry, Christian-Albrechts-Universität, \\ D-24098 Kiel, Germany \\ ${ }^{2}$ Institute of Animal and Aquacultural Sciences, Agriculture University of Norway, \\ Box 5052, 1432 Ås, Norway
}

\section{ABSTRACT}

The estimation of the expected loss of genetic diversity and marginal diversities in a set of breeds within a defined future time horizon requires initial estimates of breed extinction probabilities. In this study, the extinction probabilities of 5 German dual-purpose cattle breeds were estimated by population viability analysis. Regression was used to estimate the infinitesimal mean and variance of the population growth and this was based on the diffusion approximation of the density independent population growth (also known as the Dennis regression model). The annual number of milk-recorded cows in each breed was used as census data. Based on the regression results, the extinction probabilities and their confidence intervals were estimated for a wide variety of future time horizons using Monte Carlo time series simulations. The estimates of extinction probabilities were sensible, but in 2 cases they depended heavily on the time horizon considered. Additionally, the confidence intervals became very wide with an increased time horizon. We recommend the estimation of extinction probabilities for a set of future time horizons rather than for a single future time and the selection of an upper bound for this set that is not too large to be meaningful. The validity of the use of the number of milk-recorded cows as census data and of the model assumptions is discussed in detail.

(Key words: extinction probability, population viability analysis, livestock breed, genetic diversity)

Abbreviation key: $\mathbf{C I}=$ confidence interval, PVA = population viability analysis.

\section{INTRODUCTION}

A main reason for the loss of genetic diversity in livestock species is the permanent extinction of breeds

Received February 21, 2005

Accepted April 12, 2005.

Corresponding author: Jörn Bennewitz; e-mail: jbennewitz@ tierzucht.uni-kiel.de.
(FAO, 2000). To stop this loss, all endangered breeds would ideally be conserved, but because of financial restraints, it is only possible to establish a diversity conservation plan that includes those breeds considered relevant for the diversity. The prerequisite for the identification of those breeds is the application of a suitable diversity measure together with probabilities of extinction of the breeds (Simianer et al., 2003).

Two marker-assisted and kinship-based (Eding and Meuwissen, 2001) core set diversity measures, 1) the maximum variance offspring core set diversity, and 2) the maximum variance total core set diversity, are now available, and are especially appropriate to describe the diversity on a breed level (Eding et al., 2002; Bennewitz and Meuwissen, 2005). The maximum variance offspring and maximum variance total core sets are built by relative breed contributions to maximize the variance of putative offspring from interbred conserved breeds and to maximize the total genetic variance that can be found within and between conserved breeds, respectively (Eding et al., 2002; Bennewitz and Meuwissen, 2005). Within these 2 core set approaches, a ranking of breeds for inclusion in a conservation plan can be conducted according to their relative contribution to the core set. However, these relative contributions are valid only for a situation where all breeds are alive. To draw inference from the present diversity on expected future diversity, the extinction probabilities of the breeds have to be taken into account. Weitzman (1993) and Simianer et al. (2003) showed how extinction probabilities can be combined with a diversity measure in order to estimate the expected future diversity and hence the expected loss of diversity. Additionally, this strategy allows the estimation of the marginal diversity of a breed, which is defined as the change of the diversity at the end of a defined time horizon when the extinction probability of the breed would be changed by one unit (Simianer et al., 2003). Simianer et al. (2003) presented a sophisticated method showing how the marginal diversities of the breeds can be used to identify the most efficient genetic diversity conservation scheme. 
Hence, although suitable diversity measures are available for livestock breeds, until now little has been known about the determination of their extinction probabilities. To our knowledge, only Reist-Marti et al. (2003) have estimated extinction probability in livestock breeds. Reist-Marti et al. (2003) assigned relative values to 10 different breed-specific variables and defined the extinction probability of a breed as the sum of the values that the particular breed obtained. The method of Reist-Marti et al. (2003) has a pioneering character and because of its comprehensiveness, it seems to be appealing. However, a drawback is that it does not produce any standard errors or confidence intervals (CI) of the estimates. As pointed out by various authors (e.g., Ludwig, 1999; Fieberg and Ellner, 2000; Ellner et al., 2002; Reed et al., 2002), extinction probability estimates should always be reported with their respective CI because these can become very wide (often covering the whole parameter space), especially when the time horizon is large or the amount of data is sparse. This result occurs regardless of the method for estimation. In addition, the approach of ReistMarti et al. (2003), involving somewhat arbitrary assignment of the relative values to the variables, cannot be considered completely satisfactory.

In contrast to livestock breeds, the estimation of extinction properties for endangered wildlife species is frequently conducted by conservation biologists, with population viability analysis (PVA) as the main statistical tool (e.g., Soule, 1993; Burgman et al., 1996; Beissinger and Westphal, 1998). A wide variety of PVA methods was developed to cope with the different situations (e.g., different availability of data) that can be found in wildlife species (see previously listed authors). One of the main PVA models used is the population growth diffusion approximation model of Lande and Orzack (1988) and Dennis et al. (1991).

The aim of the present study was to use the PVA diffusion approximation model of Lande and Orzack (1988) and Dennis et al. (1991) for the estimation of extinction probabilities in livestock breeds. The regression approach of Dennis et al. (1991) was used to estimate population growth parameters for 5 German dual-purpose cattle breeds with numbers of milk-recorded cows as observations for the population size. The extinction probabilities were estimated by MonteCarlo simulations using formulae of Dennis et al. (1991).

\section{MATERIALS AND METHODS}

This section is divided into 4 subsections. First, the applied PVA model is described briefly. Detailed descriptions, including an exhaustive literature review, can be found in Lande and Orzack (1988) and Dennis et al. (1991). Next, a short description of the estimation of population growth parameters is given. The estimation of extinction parameters is described in detail. Finally, the information regarding the specific breeds and data sets used in this study are presented.

\section{The PVA Population Growth Diffusion Approximation Model}

Assume the following density independent growth model in an age-structured population

$$
\mathbf{n}(t+1)=\mathbf{A}(t) \mathbf{n}(t),
$$

where $\mathbf{n}$ is a column vector that contains the number of female individuals in each age class for each time $t(t=0,1,2, \ldots, q)$. $\mathbf{A}$ is a square stochastic transition matrix and is defined for each time $t$. $\mathbf{A}$ is also known as the Lesli matrix and contains age-specific fecundity rates in the top row, age-specific survival probabilities along the sub-diagonal, and zeros elsewhere. The stochasticity of $\mathbf{A}$ is due to a fluctuating environment. From the set of known A matrices, it is possible to estimate the infinitesimal mean $\mu$ (known as the longrun growth rate of the population, it describes the rate at which the median log population size increases over time) and the infinitesimal variance $\sigma^{2}$ using equations of Lande and Orzack (1988) and Dennis et al. (1991). The parameters $\mu$ and $\sigma^{2}$ are of fundamental importance because the calculation of a number of population growth parameters and population extinction property estimates from these 2 parameters is straightforward (as shown below). However, the reliable estimation of the numerous elements in the various A matrices might be difficult, which makes the direct estimation of $\mu$ and $\sigma^{2}$ from A problematic. Fortunately, the stochasticity of the $\mathbf{A}$ matrices allows under certain circumstances (see discussion section) the approximation of the population growth by a diffusion process (Lande and Orzack, 1988; Dennis et al., 1991). Using the notation of Dennis et al. (1991), $N(t)$ is the population size at time $t$ and $X(t)=\log [N(t)]$. Based on the central limit theorem, for a large $t, X(t)$ becomes approximately normally distributed as follows

$$
X(t) \sim N\left(x_{0}+\mu t, \sigma^{2} t\right),
$$

where $x_{0}$ is the log of the first observed population size $\left(n_{0}\right)$. Because of the normal distribution of $X(t)$, it can be modeled by a Wiener drift process with $\mu$ as the infinitesimal mean and $\sigma^{2}$ as the infinitesimal variance of the process (Lande and Orzack, 1988; Dennis et al., 
1991). This property enables the possibility to estimate $\mu$ and $\sigma^{2}$ from time series census data by a linear regression without setting up the A matrices (Dennis et al., 1991).

\section{Linear Regression and Estimation of Population Growth Parameters}

Based on this population growth diffusion approximation model (Dennis et al., 1991), in the present study, linear regression was performed to estimate $\mu$ and $\sigma^{2}$ for the cattle data described below. Assume that the number of individuals of a population was observed at different times $t_{0}, t_{1}, \ldots, t_{q}$ with observed figures $n\left(t_{0}\right)=n_{0}, n\left(t_{1}\right)=n_{1}, \ldots, n\left(t_{q}\right)=n_{q}$. The time interval between 2 observations was $t_{1}-0=\tau_{1}, t_{2}-t_{1}=$ $\tau_{2}, \ldots, t_{q}-t_{q-1}=\tau_{q}$. The dependent variable was defined for each $i(i=1,2, \ldots, q)$ as $y_{i}=\left[\log \left(n_{i} / n_{i-1}\right)\right] / \sqrt{\tau_{i}}$ and was regressed on $\sqrt{\tau_{i}}$ without an intercept. The regression coefficient yields an estimate for $\mu$ and the residual variance an estimate for $\sigma^{2}$, denoted as $\hat{\mu}$ and $\hat{\sigma}^{2}$, respectively.

The following equations, [2] to [10], were applied to the cattle breed data and were taken from Dennis et al. (1991). They describe the estimation of population growth parameters from $\hat{\mu}$ and $\hat{\sigma}^{2}$. The distributions of the 2 parameter estimates $\hat{\mu}$ and $\hat{\sigma}^{2}$ are

$$
\begin{gathered}
\hat{\mu} \sim N\left(\mu, \sigma^{2} / t_{q}\right) \text { and } \\
(q-1) \hat{\sigma}^{2} / \sigma^{2} \sim \chi_{q-1}^{2}, \text { respectively. }
\end{gathered}
$$

The CI of $\mu$ with a defined Type-I-error-rate of $\alpha$ was estimated as

$$
\hat{\mu}-t_{\alpha / 2, q-1} \sqrt{\hat{\sigma}^{2} / t_{q}}, \hat{\mu}+t_{\alpha / 2, q-1} \sqrt{\hat{\sigma}^{2} / t_{q}},
$$

where $t_{\alpha / 2, q-1}$ denotes the $100(1-\alpha / 2)$ percentile of a $t$ distribution with $q-1$ degrees of freedom. The central CI for $\sigma^{2}$ with a defined $\alpha$ was estimated as

$$
(q-1) \hat{\sigma}^{2} / \chi_{\alpha / 2, q-1}^{2},(q-1) \hat{\sigma}^{2} / \chi_{1-\alpha / 2, q-1}^{2} .
$$

From $\hat{\mu}$ and $\hat{\sigma}^{2}$ the continuous rate of increase $(r)$ and the finite rate of increase $(\lambda)$ were estimated as

$$
\hat{\lambda}=\exp (\hat{r})=\exp \left[\hat{\mu}+\left(\hat{\sigma}^{2} / 2\right)\right] .
$$

The finite rate of increase $\lambda$ describes the proportion by which the population changes on average every time step; $\lambda$ is also sometimes termed discrete rate of increase. The CI for a defined $\alpha$ was estimated as

$$
\exp \left\{\hat{r} \pm z_{\alpha / 2} \sqrt{\hat{\sigma}^{2}\left[\frac{1}{t_{q}}+\frac{\hat{\sigma}^{2}}{2(q-1)}\right]}\right\},
$$

where $z_{\alpha / 2}$ is the $100[1-(\alpha / 2)]$ th percentile of the standard normal distribution. The CI of $r$ was the log of the corresponding CI of $\lambda$.

Further, the expected population size on a log scale at a defined future time $t_{f}\left(t_{f}>t_{q}\right)$ was estimated from $\hat{\mu}$

$$
E\left[X\left(t_{f}\right) \mid X\left(t_{q}\right)=x_{q}\right]=x_{q}+\hat{\mu} s,
$$

where $s=t_{f}-t_{q}$ and $t_{f}$ was varied as $t_{f}=t_{q}+1, \ldots, t_{q}$ +250 in this study. The forecasted median population size on a normal scale was estimated as

$$
\operatorname{Med}\left[N\left(t_{f}\right)\right]=\exp \left\{E\left[X\left(t_{f}\right) \mid X\left(t_{q}\right)=x_{q}\right]\right\} .
$$

The median population size was used in this study for forecasting because it describes the diffusion approximation better than the mean or expected population size (Dennis et al., 1991). The estimation of the CI of $X\left(t_{f}\right)$ for a defined $\alpha$ was straightforward:

$$
E\left[X\left(t_{f}\right) \mid X\left(t_{q}\right)\right]=x_{q} \pm t_{\alpha / 2, q-1} \sqrt{\hat{\sigma}^{2} s\left(1+\frac{s}{t_{q}}\right)} .
$$

The CI for forecasted $\operatorname{Med}\left[N\left(t_{f}\right)\right]$ was obtained by performing an exponential transformation to the lower and upper bounds of the CI from [10], respectively.

\section{Estimation of Extinction Probability}

Before extinction probabilities can be estimated, the term population extinction has to be defined. Obviously, a population is extinct if its last individual has died. In general, it is more useful to define a critical threshold $\left(n_{c}\right)$ for the number of individuals and call a population quasi-extinct if its population size falls below this threshold. The term quasi-extinction was introduced in this context by Ginzburg et al. (1982) and can be interpreted as a policy threshold that indicates the need of intervention to maintain the breed. The choice of $n_{c}$ can be made according to different aspects (Gandini et al., 2004) and is always arbitrary to some extent. In this study, 2 critical values were used, $n_{c}=1000$ females and $n_{c}=100$ females, respectively, which were based on the arguments given by Gandini et al. (2004). According to Gandini et al. (2004), the lower bound of self-sustainability of a livestock breed is around 1000 females. Additionally, without any conservation effort, a breed size of below 100 females increases the extinction process rapidly, e.g., 
due to low number of herds or low economical competitiveness of the breed.

If the long-run population growth rate for a breed or species is $\leq 0$, one can be certain that the population will become extinct at a future point in time, assuming no intervention is done. However, even when the growth rate is positive, a chance of extinction exists due to random fluctuations of population size. Let us define $x_{d}$ as the distance on the log scale between the last observed population size $\left(n_{q}\right)$ and the threshold, $x_{d}=x_{q}-x_{c}=\log \left(n_{q} / n_{c}\right)$. The probability $(\pi)$ that a population will ever become extinct is (Dennis et al., 1991)

$$
\pi=\left\{\begin{array}{l}
1, \mu \leq 0 \\
\exp \left(-2 \mu x_{d} / \sigma^{2}\right), \mu>0
\end{array}\right\} .
$$

The probability of extinction (i.e., crossing the defined threshold $n_{c}$ ) can be calculated for every future point of time. For conservation efforts, it is of more interest to estimate the cumulative probability of extinction for the future points of time within a time span $t_{e}$. For a defined $t_{e}$ (e.g., $t_{e}=50$ years) the cumulative extinction probability, i.e., the probability that the population size will cross the threshold $n_{c}$ at least one time, is denoted in the following as simply the extinction probability $\left(\pi_{t_{e}}\right)$. It is defined as

$$
\begin{gathered}
\pi_{t_{e}}=\pi *\left[\Phi\left(\frac{-x_{d}+|\mu| t_{e}}{\sigma \sqrt{t_{e}}}\right)\right. \\
\left.+\exp \left(2 x_{d}|\mu| / \sigma^{2}\right) \Phi\left(\frac{-x_{d}-|\mu| t_{e}}{\sigma \sqrt{t_{e}}}\right)\right]
\end{gathered}
$$

where $\Phi(\cdot)$ denotes for the standard normal cumulative distribution function. Equation [12] is presented by Dennis et al. (1991) without $\pi$, which corresponds to the conditional extinction probability (conditional on the fact that the population is sure to eventually become extinct, $\pi=1$ ). However, also breeds with a positive long run population growth (i.e., $\mu>0$ and subsequently $\pi<1$ ) show a positive extinction probability due to random fluctuations (as mentioned above). By incorporating $\pi$ in [12], it is possible to define the unconditional extinction probability and subsequently to define the extinction probability for breeds with a positive $\mu$.

In principle, it is possible to estimate $\pi$ and $\pi_{t_{e}}$ using equations [11] and [12], respectively, by replacing $\mu$ and $\sigma^{2}$ with respective estimates obtained from the regression. However, this would not make use of the distribution of the parameter estimates as shown in
[2] and [3]. To account for this variation, $\pi$ and $\pi_{t_{e}}$ were estimated in this study by a Monte Carlo time series simulation. The simulation is based on the approximate normal distribution of $X(t)$ as shown in [1]. A number of $S$ time series of log population sizes was simulated, each of exactly the same size and same time of observation as the original data set. At the beginning of each time series simulation $s$, the first log population size $\left(x_{s, 0}\right)$ was set to the original first observed log population size, i.e., $x_{s, 0}=x_{0}$. Each next $\log$ population size $x_{s, i}(\mathrm{i}=1, \ldots, q)$ was sampled from

$$
x_{s, i} \sim N\left(x_{s, i-1}+\hat{\mu} \tau_{i}, \tau_{i} \hat{\sigma}^{2}\right),
$$

where $\tau_{i}$ is the same as defined for the regression (defined above). The $S$ simulated time series were analyzed by the regression as described above, which yielded $S$ different pairs of $\mu$ and $\sigma^{2}$, denoted as $\hat{\mu}_{s}$ and $\hat{\sigma}_{s}^{2}$, respectively. The pairs of $\hat{\mu}_{s}$ and $\hat{\sigma}_{s}^{2}$ were inserted into [11] in order to obtain $S$ different $\hat{\pi}_{s}$. Next, the $S$ pairs of $\hat{\mu}_{s}, \hat{\sigma}_{s}^{2}$ and $\hat{\pi}_{s}$ were used in [12] to obtain $S$ different $\hat{\pi}_{s, t_{e}}$. Finally, the mean of all $\hat{\pi}_{s}$ and $\hat{\pi}_{s, t_{e}}$ were taken as the estimates for $\pi$ and $\pi_{t_{e}}$, respectively. Using this approach, the estimation of a CI for the extinction property parameters was straightforward. The upper and lower bounds of $95 \% \mathrm{CI}$ of $\pi$ and $\pi_{t_{e}}$ were calculated by taking the top and bottom 2.5th percentile of the distribution of the $S \hat{\pi}_{s}$ and $\hat{\pi}_{s, t}$, respectively. A similar method was used by Dennis and Otten (2000). The $S$ was chosen to be 2000 and $t_{e}$ was varied between 1 and 250 in this study.

\section{Cattle Breeds}

Data from 5 German dual-purpose cattle breeds were analyzed by the methods described above. No census data reporting the number of total population size was available for the breeds, but the numbers of milk-recorded cows have been available for many years (Figure 1) and these were used in this study as observations. All breeds are listed in the animal genetic database of the European Association for Animal Production (EAAP, 2005). A short description of the breeds is given; for more details see the EAAP database entries. All breeds are supervised in some form by breeding organizations.

The Angler breed is a dual-purpose red cattle breed with a primary focus on milk production. It belongs to the central European red group of cattle breeds and is found mainly in the northern part of Germany, in Schleswig-Holstein. Since the 1960s, genetics from other breeds have been introduced and the original 

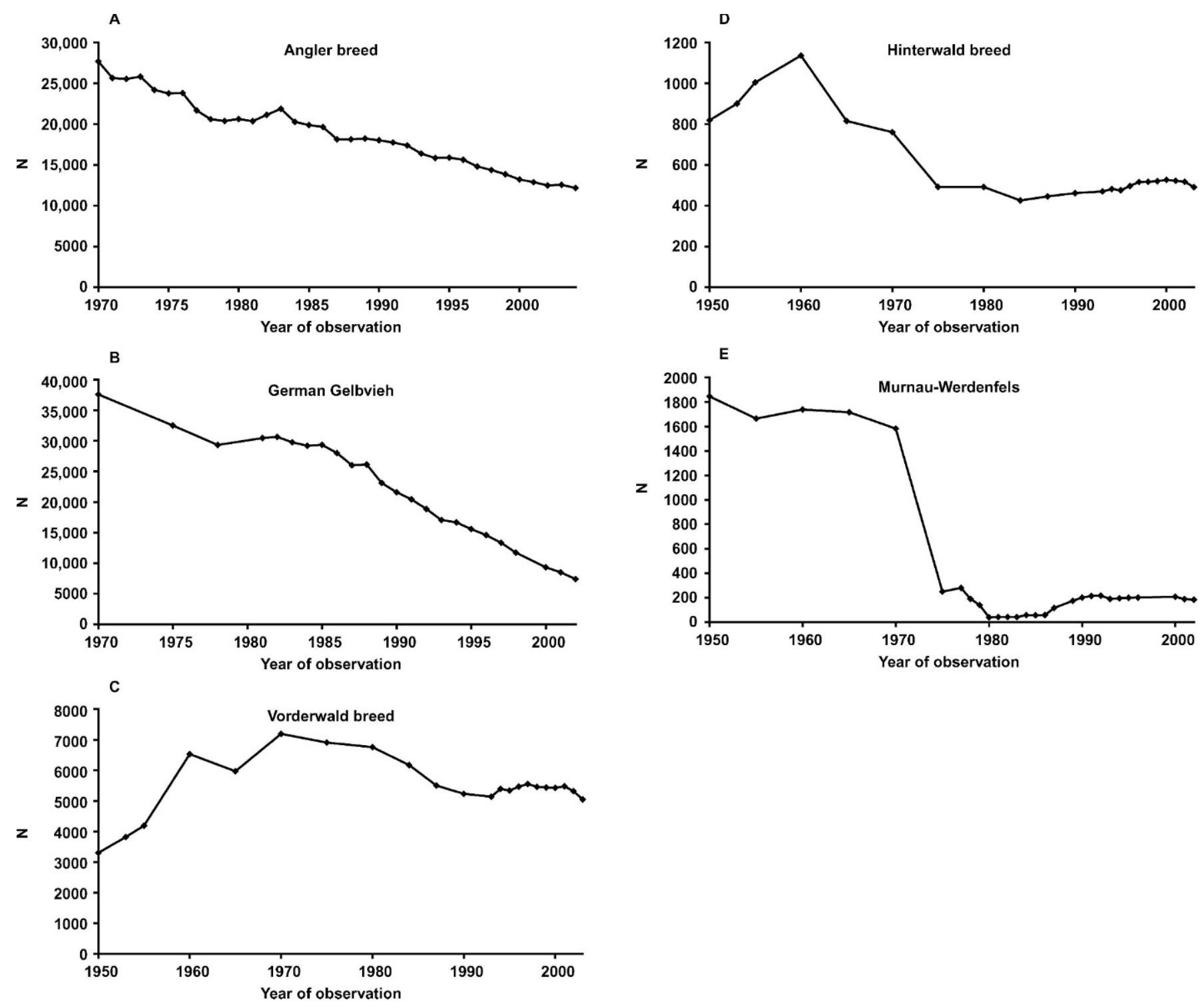

Figure 1. Number of observed milk-recorded cows and year of observation for the 5 breeds.

Angler breed is very close to final extinction (only very few cows are still alive). The number of milk-recorded Angler cows in Schleswig-Holstein has been decreasing for many years (Figure 1).

The German Gelbvieh belongs to the blond cattle group and is a beef-type dual-purpose breed. Its main location is in the southern part of Germany, in Franconia, although it has also been used purely for meat production in the Eastern part of Germany since the beginning of the last decade. Since the middle of the last century, genetics from other breeds have been introduced. The number of milk-recorded German Gelbvieh cows in Germany over the past decades is decreasing steadily (Figure 1 ).
The Vorderwald breed is an old local breed with its main location in the southern part of Germany, in the southern Black Forest. It belongs to the red pattern group and is a dairy type dual-purpose breed. Genetics from other breeds have been introduced since the middle of the last century. Since recently, it is also used for landscape management. The number of females from milk-recording data from the main location of the breed from the last decades increased from 1950 to 1970 but have since then been slightly decreasing (Figure 1).

Similarly, the Hinterwald breed is an old native breed located in the southern part of Germany, in the southern Black Forest, and belongs to the red pattern 
Table 1. First $\left(n_{0}\right)$ and last $\left(n_{q}\right)$ observed population size (number of cows milk-recorded), number of transitions $(q)$, time span in years between first and last observation $\left(t_{q}\right)$, and source of observation.

\begin{tabular}{lrrlll}
\hline Breed & \multicolumn{1}{l}{$n_{0}$} & $n_{q}$ & $q$ & $t_{q}$ & Source $^{1}$ \\
\hline Angler & 27,674 & 12,175 & 34 & 34 & 1 \\
German Gelbvieh & 37,574 & 7419 & 23 & 32 & 2 \\
Vorderwald & 3301 & 5048 & 21 & 53 & 3 \\
Hinterwald & 819 & 491 & 21 & 53 & 3 \\
Murnau-Werdenfels & 1845 & 184 & 27 & 52 & 2,4 \\
\hline
\end{tabular}

${ }^{1}$ Sources: 1 = Landeskontrollverein Schleswig-Holstein e.V., Kiel, Germany; 2 = Arbeitsgemeinschaft Deutscher Rinderzüchter e.V., Bonn, Germany; 3 = Rinderunion Baden Württemberg e.V., Stuttgart, Germany; and 4 = Bayrische Landesastalt für Landwirtschaft, Grub, Germany.

group. Genetics from other breeds have been introduced into this breed from the Vorderwald breed since the middle of the last century. This breed is used for production of milk and meat and for landscape management. The number of females from milk-recording data from the main location of the breed decreased significantly between 1960 and 1975 but since then it has been more or less stable (Figure 1).

The Murnau-Werdenfels is a small autochthonous local dual-purpose breed with its main location in the southern part of Germany, in the Werdenfels Land in Bavaria. It belongs to the blond group of cattle breeds. The numbers of cows from milk-recording data from Germany over the last decades are shown in Figure 1 . For additional figures used in the regression analysis, see Table 1.

\section{RESULTS}

The estimated population growth parameters and the forecasted median population sizes are presented in Table 2 and Figure 2, respectively. Due to the continuously decreasing past population size of the Angler and German Gelbvieh, these breeds had negative $\hat{\mu}$ and the upper CI bounds of $\hat{\mu}$ were negative. Additionally, $\hat{\sigma}^{2}$ was small. Hence, $\hat{r}$ was negative, and the forecasted median population size together with the corresponding CI were strongly decreasing with increasing future time for these 2 breeds. For the Vorderwald breed, $\hat{\mu}$ and $\hat{r}$ were both slightly positive and for the Hinterwald breed, both were slightly negative. The CI of $\hat{\mu}$ and $\hat{r}$ included the value zero for both the Vorderwald and Hinterwald breeds, and the lower and upper bounds of the CI of the forecasted median population sizes were diverging with increasing future time for these 2 breeds, which makes the estimation very uncertain with increasing future time. The MurnauWerdenfels showed a negative $\hat{\mu}$ but a positive $\hat{r}$. This discrepancy of $\hat{\mu}$ and $\hat{r}$ is due to the relatively large $\hat{\sigma}^{2}$ for this breed (Eq. 6). Therefore, despite the positive $\hat{r}$, the forecasted median population size decreased

Table 2. Estimated population growth parameters and results from the Durbin-Watson test for the breeds. The upper and lower bounds of the growth parameter $95 \%$ confidence interval are shown in italics.

\begin{tabular}{lllllll}
\hline Breed & $\hat{\mu}^{1}$ & $\hat{\sigma}^{22}$ & $\hat{r}^{3}$ & $\hat{\lambda}^{4}$ & $\mathrm{DW}^{5}$ & $\mathrm{P}_{\mathrm{DW}^{6}}$ \\
\hline Angler & -0.024 & 0.001 & -0.024 & 0.977 & 1.974 & 0.469 \\
& -0.013 & 0.002 & -0.013 & 0.987 & & \\
German Gelbvieh & -0.035 & 0.001 & -0.034 & 0.966 & & 0.927 \\
& -0.051 & 0.003 & -0.049 & 0.952 & 0.970 \\
Vorderwald & -0.031 & 0.006 & -0.031 & 0.970 & & \\
& -0.070 & 0.002 & -0.068 & 0.935 & & \\
& 0.008 & 0.004 & 0.010 & 1.010 & 1.611 & 0.181 \\
Hinterwald & 0.025 & 0.007 & 0.026 & 1.026 & & \\
& -0.009 & 0.002 & -0.006 & 0.994 & & \\
& -0.010 & 0.004 & -0.008 & 0.992 & 1.754 & \\
Murnau-Werdenfels & 0.009 & 0.008 & 0.010 & 1.010 & & \\
& -0.028 & 0.002 & -0.025 & 0.975 & \multirow{2}{*}{1.667} & 0.191 \\
& -0.044 & 0.123 & 0.017 & 1.017 & & \\
\hline
\end{tabular}

${ }^{1}$ Estimated diffusion process drift parameter (long-run population growth).

${ }^{2}$ Estimated diffusion process variance parameter.

${ }^{3}$ Estimated continuous rate of population increase.

${ }^{4}$ Estimated finite rate of population increase.

${ }^{5}$ Durbin-Watson test statistic.

${ }^{6}$ Error probability of Durbin-Watson test statistic for positive first-order autocorrelation (the error probability for negative first-order autocorrelation was always higher). 

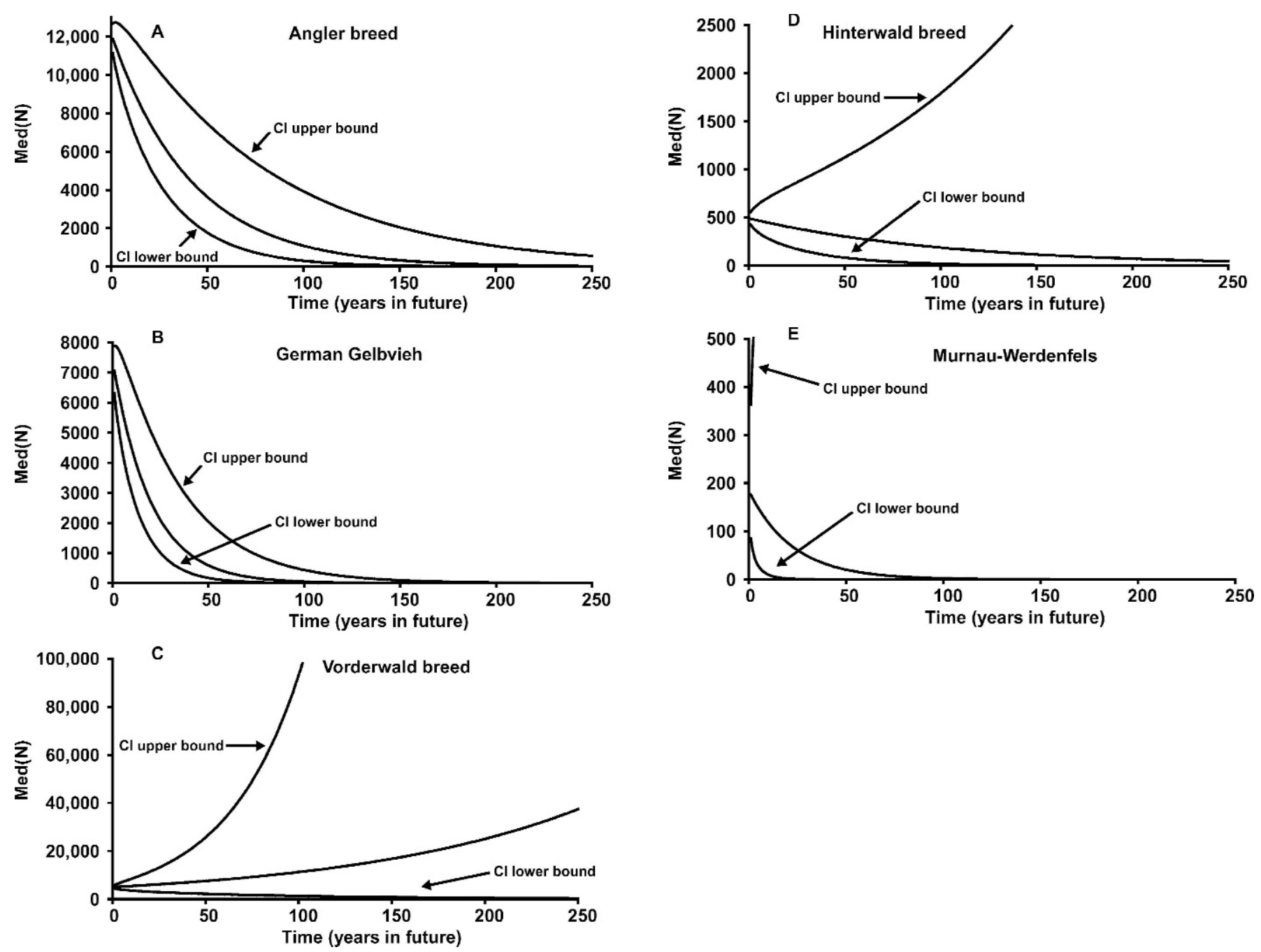

Figure 2. Forecasted median population size $[\operatorname{Med}(\mathrm{N})]$ of the 5 breeds plotted against the future time in years. Additionally the upper and lower bounds of the 95\% confidence interval (CI upper bound and CI lower bound, respectively) are presented.

quickly with increasing future time, but the corresponding CI for the median population sizes were very wide. The estimates of the finite rate of increase $(\hat{\lambda})$ are listed in Table 2 without mention here because they are solely a function of $\hat{r}$ : if $\hat{r}$ was negative, $\hat{\lambda}$ was below one and if $\hat{r}$ was positive, $\hat{\lambda}$ was above one (Eq. 6 ).

The plots of the extinction probabilities $\left(\hat{\pi}_{t_{e}}\right)$ of the breeds against the time span considered $\left(t_{e}=1, \ldots\right.$, 250) are shown in the Figures 3 to 7. For the Angler breed, $\hat{\pi}_{t_{e}}$ was nearly zero for the next 60 (120) years and $n_{c}=1000\left(n_{c}=100\right)$, increased strongly from that point onward and quickly reached a value close to one (Figure 3). The plot of $\hat{\pi}_{t_{e}}$ for the German Gelbvieh follows a similar pattern (Figure 4). It was almost zero for the first $30(60)$ years for $n_{c}=1000\left(n_{c}=100\right)$ and increased rapidly to one during the following decades.
Compared with the Angler breed, this increase was much stronger. For both breeds, the CI of $\hat{\pi}_{t_{e}}$ covered almost the whole parameter space if $\hat{\pi}_{e}$ was above zero and below one. In contrast, if $\hat{\pi}_{t_{e}}$ was approximately zero or approximately one, the CI width was nearly zero for both breeds (Figure 3 and 4).

The Vorderwald breed showed a $\hat{\pi}_{t_{e}}$ below 0.02 for the next $100 \mathrm{yr}$ and below 0.01 for the entire time span considered, regardless of $n_{c}$ (Figure 5 , note the scaling of the y axis). For $n_{c}=1000, \hat{\pi}_{t_{e}}$ started to increase slowly and almost linearly for $t_{e}>50 \mathrm{yr}$. The lower bound of the CI was always zero. The upper bound started to increase strongly at $t_{e}=50$ years $\left(t_{e}=200\right)$ for $n_{c}=1000\left(n_{c}=100\right)$, making the interval width wide for larger $t_{e}$. 

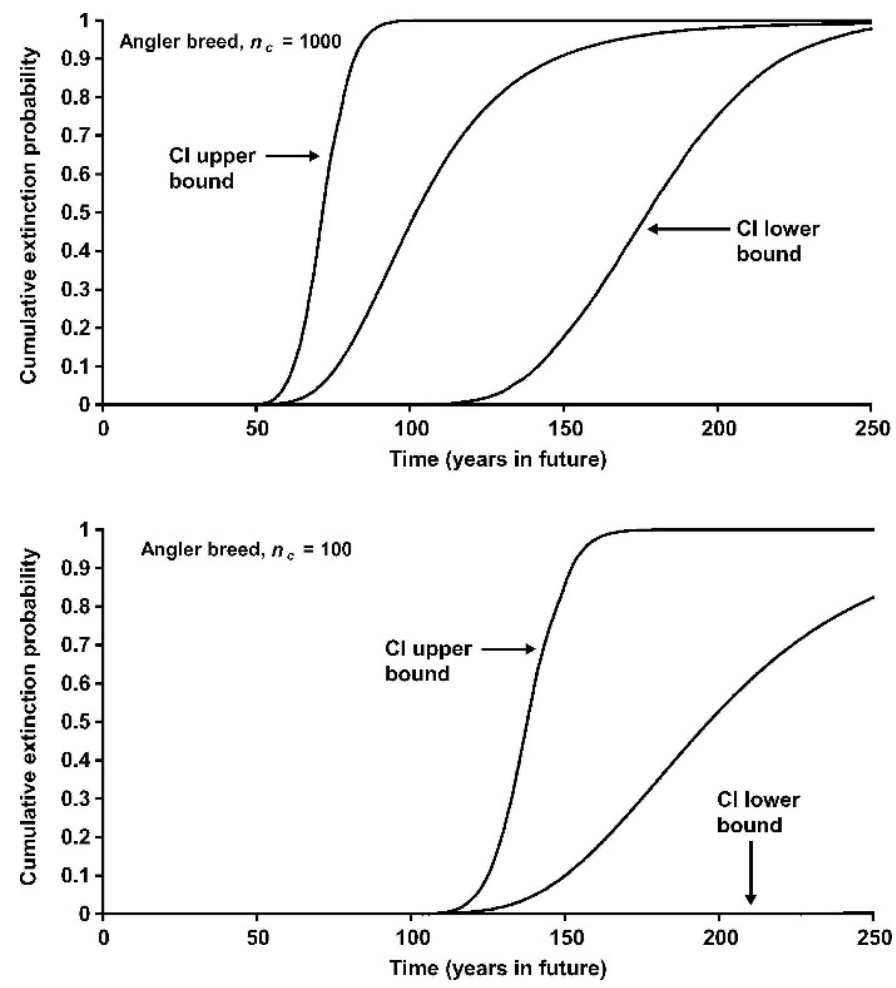

Figure 3. Cumulative extinction probability $\left(\hat{\pi}_{t}\right)$ of the Angler breed plotted against the future time ( $t_{e}$ in years) for a lower critical threshold $n_{c}=1000$ (top) and $n_{c}=100$ (bottom). Additionally the upper and lower bounds of the 95\% confidence interval (CI upper bound and CI lower bound, respectively) are presented. Note that for $n_{c}=100$ the lower bound of the 95\% CI is always close to zero and therefore the corresponding line is almost identical with the $\mathrm{x}$-axis.

For the Hinterwald breed as well as for the MurnauWerdenfels, $\hat{\pi}_{t_{e}}$ could only be estimated for $n_{c}=100$, because the last observed population size was below 1000. If $n_{c}=1000$ had been considered as the lower critical threshold for extinction, both breeds would already have been extinct. For the Hinterwald breed, $\hat{\pi}_{t_{e}}$ was almost zero for the next $30 \mathrm{yr}$ but at this point, it started to increase (Figure 6). The lower bound of the CI was zero for every considered $t_{e}$, but the upper bound increased quickly from nearly zero at $t_{e}=25$ to nearly one at $t_{e}=80$. Hence, for every considered $t_{e}>$ 80 , the CI spanned almost the whole parameter space. The Murnau-Werdenfels is the most endangered breed (Figure 7) due to the small last observed population size and the relatively large $\hat{\sigma}^{2}$. Its $\hat{\pi}_{t_{e}}$ increased from 0.1 to around 0.7 within $t_{e}=1$ and $t_{e}=20$ and stayed remarkably constant near 0.85 with only a small increase for $t_{e}>40$. The upper CI bound of $\hat{\pi}_{t_{e}}$ quickly reached one and the lower bound increased rapidly for $t_{e}<30$ and from then on it remained remarkably
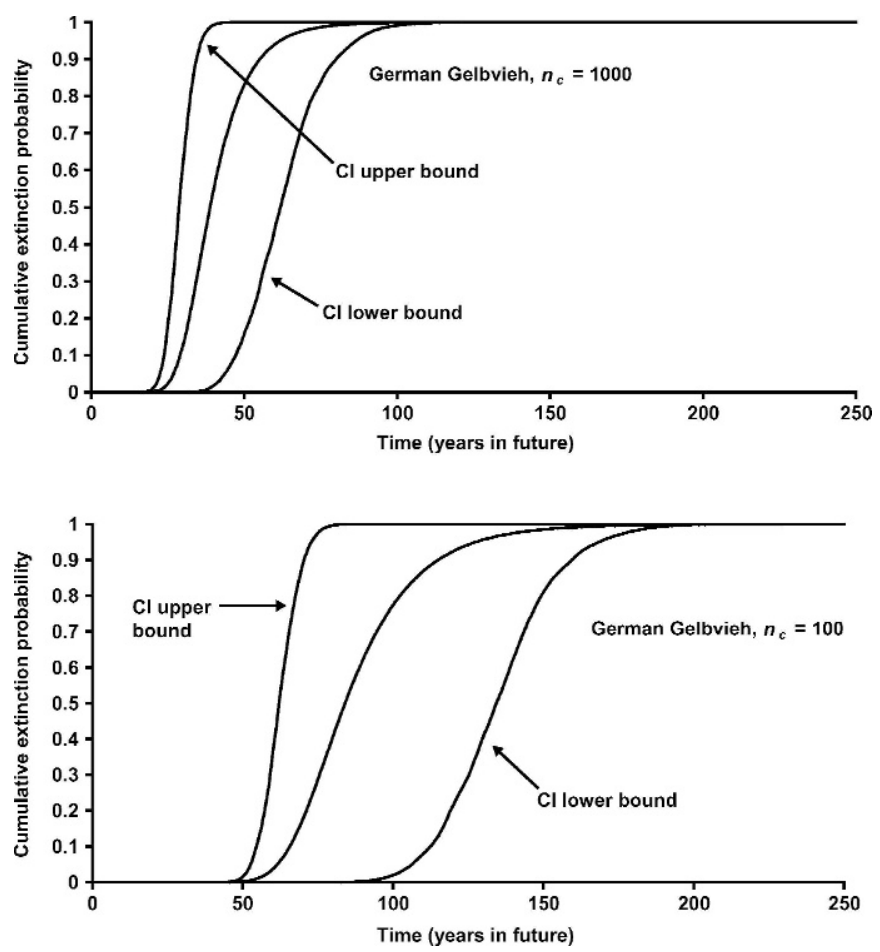

Figure 4. Cumulative extinction probability $\left(\hat{\pi}_{t_{e}}\right)$ of the German Gelbvieh plotted against the future time $\left(t_{e}\right.$ in years) for a lower critical threshold $n_{c}=1000$ (top) and $n_{c}=100$ (bottom). Additionally the upper and lower bounds of the 95\% confidence interval (CI upper bound and CI lower bound, respectively) are presented.

constant at around 0.5 . The CI width was more or less constant around 0.5 for $t_{e}>30$.

\section{DISCUSSION}

The estimation of expected future genetic diversity and of the breed marginal diversities needs estimates of breed extinction probabilities. Therefore, an attempt was made to estimate the extinction probabilities from 5 German dual-purpose cattle breeds by applying a convenient PVA method using the ideas from Dennis et al. (1991). The discussion section is divided into 2 parts. In the first part, the results of the study and their appropriateness for estimation of marginal diversities and expected future diversity are discussed. The second part describes the assumptions of the applied PVA model and their validity in the present study.

\section{Extinction Probabilities}

The most informative results of the applied $\pi_{t_{e}}$ estimation procedure are the plots of $\hat{\pi}_{t_{e}}$ and their CI bounds against a series of defined future time horizons 

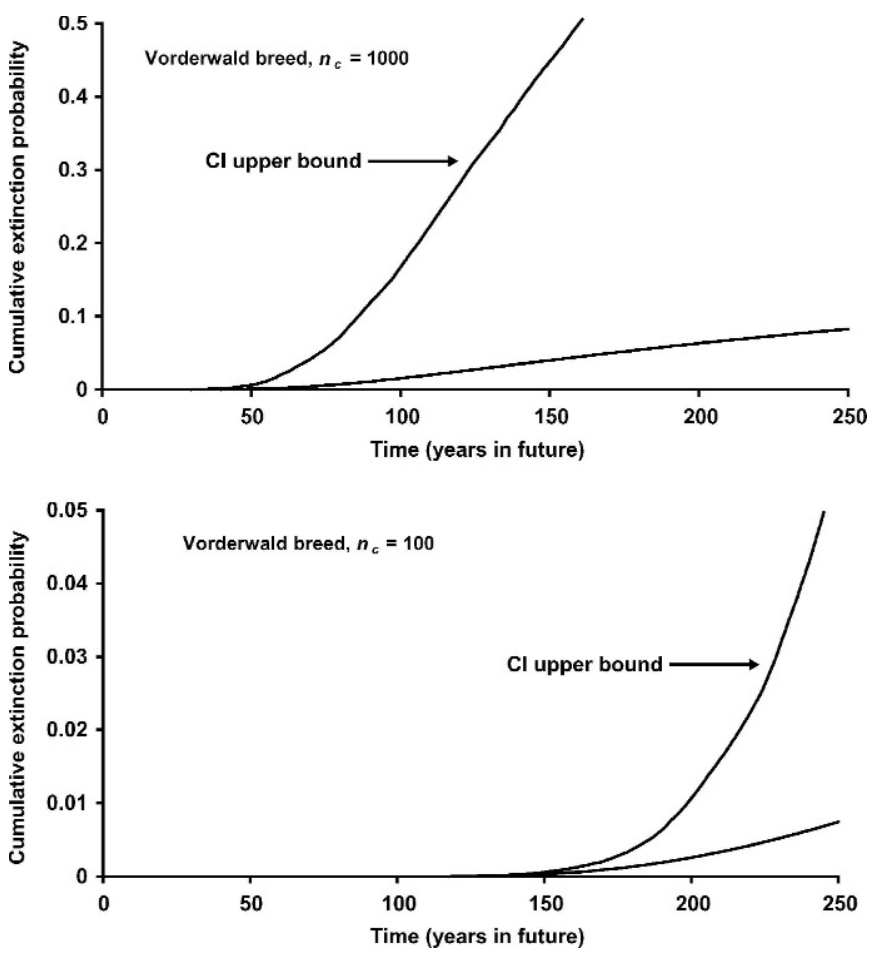

Figure 5. Cumulative extinction probability $\left(\hat{\pi}_{t}\right)$ of the Vorderwald breed plotted against the future time ( $t_{e}$ in years) for a lower critical threshold $n_{c}=1000$ (top) and $n_{c}=100$ (bottom). Additionally the upper bounds of the 95\% confidence interval (CI upper bound) are presented. Note that the lower bound of the $95 \%$ CI is always close to zero and therefore the corresponding line is identical with the $\mathrm{x}$-axis.

$t_{e}$ (Figure 3 to 7 ). The extinction probability estimation method of Reist-Marti et al. (2003) produced for each breed a $\hat{\pi}_{t_{e}}$ for one $t_{e}$ and it does not appear to be straightforward to expand their method to multiple $t_{e}$.

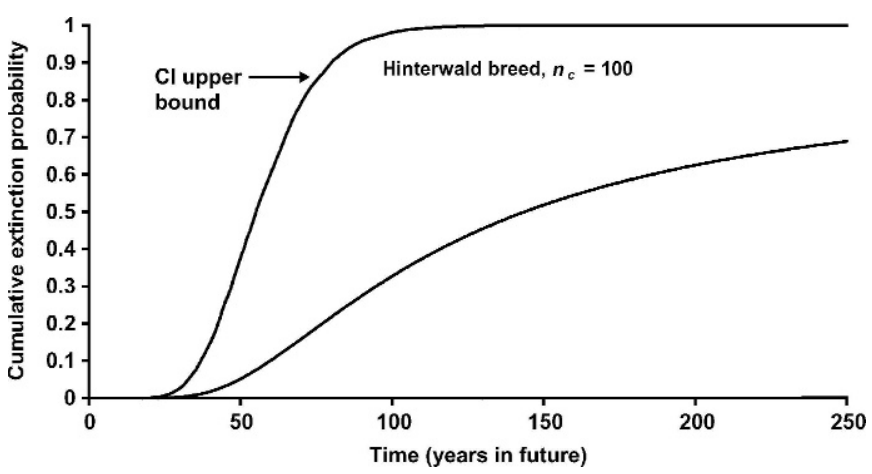

Figure 6. Cumulative extinction probability $\left(\hat{\pi}_{t}\right)$ of the Hinterwald breed plotted against the future time $\left(t_{e}\right.$ in years) for a lower critical threshold $n_{c}=100$. Additionally the upper bounds of the $95 \%$ confidence interval (CI upper bound) are presented. Note that the lower bound of the $95 \% \mathrm{CI}$ is always close to zero and therefore the corresponding line is identical with the $\mathrm{x}$-axis.

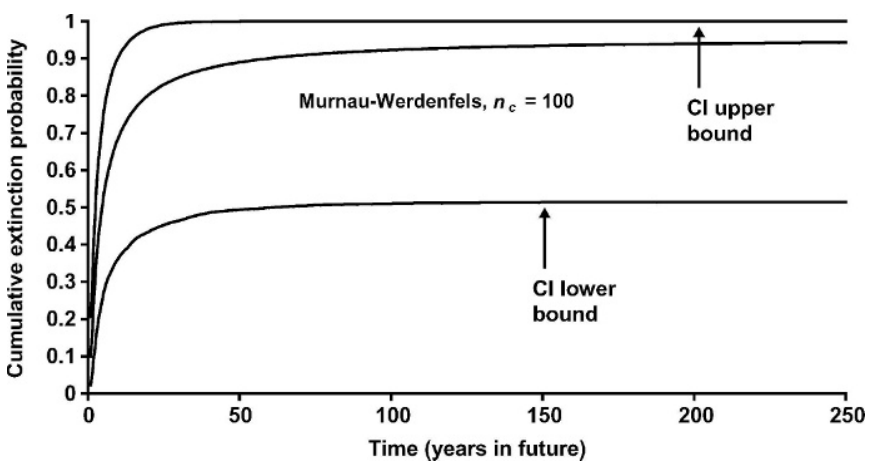

Figure 7. Cumulative extinction probability $\left(\hat{\pi}_{t_{e}}\right)$ of the MurnauWerdenfels breed plotted against the future time ( $t_{e}$ in years) for a lower critical threshold $n_{c}=100$. Additionally the upper and lower bounds of the $95 \%$ confidence interval (CI upper bound and CI lower bound, respectively) are presented.

Therefore, the expected loss of diversity was estimated only for one $t_{e}$ by Simianer et al. (2003). However, the choice of a single $t_{e}$ might be problematic if breeds such as the Angler or the German Gelbvieh are included in the experiment, which show a strong decline in population size (negative $\hat{\mu}$ ) and a small $\hat{\sigma}^{2}$, because for these breeds the time span where $\hat{\pi}_{t_{e}}$ increases from zero to one is very short. In these cases, it would be especially beneficial to use all the information that is present in the plot of $\hat{\pi}_{t_{e}}$ and to estimate an expected future diversity for every $t_{e}$ up to an upper bound of $t_{e}$, because this would provide an impression of the pattern of change of the expected loss of diversity with increasing $t_{e}$.

In this study, the upper bound of $t_{e}$ was set to be $t_{e}=$ 250 yr. For practical use this upper bound is certainly much too high, because it is very unlikely that conservation plans would be made that are based on $t_{e}$ up to $250 \mathrm{yr}$. Additionally, the reliability of $\hat{\pi}_{t_{e}}$ becomes very poor for large $t_{e}$ as shown by the CI width in Figures 3 to 7 , which suggest to estimate $\pi_{t_{e}}$ only for small tomoderate $t_{e}$. This is also strongly recommended by, for example, Beissinger and Westphal (1998), Ludwig (1999), and Fieberg and Ellner (2000). These authors showed that the choice of an appropriate $t_{e}$ is also a function of the numbers of observations and the time interval in which these were collected. The final selection of an appropriate $t_{e}$ or upper bound of $t_{e}$ remains with the researcher and is highly influenced by the time horizon of the conservation plan might be set up. Probably more important is the re-estimation of the series of $\pi_{t_{e}}$ if new observations are available, which enables a monitoring of the expected loss of diversity. In this study, the numbers of milk-recorded cows per year were used as observations - these are by-products 
of the milk-recording system. Therefore, an annual reestimation of $\pi_{t_{e}}$ and of the expected loss of diversity for a set of $t_{e}$ is possible without extra data collection effort (for further properties of numbers of milk-recorded cows as observations see the second part of the discussion section).

The level of uncertainty of $\hat{\pi}_{t_{e}}$ was estimated by time series Monte Carlo simulation. It is possible to incorporate the uncertainty of $\hat{\pi}_{t_{e}}$ in the algorithm for the expected future diversity estimation of Bennewitz et al. (J. Bennewitz, J. Kantanen, I. Tapio, M. H. Li, E. Kalm, J. Vilkki, I. Ammosov, Z. Ivanova, T. Kiselyova, R. Popov, and T. H. E. Meuwissen, 2005, unpublished data). Their algorithm can be applied repeatedly with extinction probabilities sampled from their corresponding CI assuming that each estimate within this interval is equally likely. This would produce a CI for the expected future diversity that includes the level of uncertainty of the $\hat{\pi}_{t_{e}}$.

The extinction probabilities obtained during this study are solely based on time series of population size observation. In contrast, Reist-Marti et al. (2003) obtained extinction probabilities by using breed information for 10 different variables, which seems to be a more comprehensive approach. On the other hand, it can be argued that the information regarding some of the variables used by Reist-Marti et al. (2003) is based on experiences that were made in the past and are, therefore, included here in the form of the population size time series data. For example, one variable in Reist-Marti et al. (2003) is the degree of organization of farmers. It can be assumed that if farmers were not organized, the population size would show greater amplitudes, which would result in a larger $\hat{\sigma}^{2}$. However, other factors like a putative establishment of a conservation program are not considered in the presented model. More research is needed to compare both approaches with respect to their numerical results and to find an optimal compromise between them, i.e., by combining the strengths of both approaches in some formula.

\section{PVA Model Assumptions}

Shaffer (1993) divided the factors that affect a population dynamics into the following 4 random variables: environmental fluctuations, demographic fluctuations, catastrophic events, and genetic uncertainty. The applied PVA model considers only environmental fluctuations (Lande and Orzack, 1988; Dennis et al., 1991). In the following, these 4 variables and their treatment in this study are described.
Random environmental fluctuations in wildlife species are due to unpredictable changes in the abiotic and biotic factors in the habitat of a population. This can be due to changed weather conditions (more or less rainfall or drought), food availability, competing populations, predators, parasites, etc. They might vary within a relatively short period (e.g., yearly changes in the weather conditions). The main force for the population dynamics of the breeds included in this study is their economical competitiveness in relation to the 3 large breeds, Holstein-Friesian, Simmental, and Brown Swiss. Additional factors might be fluctuations in number of herds, age of farmers, increased average milk yield per cow, together with milk quota system, diseases, in vivo conservation efforts, or sometimes, how fashionable the breed is. It is assumed that these forces are of a random environmental nature. However, compared with the environmental fluctuations in wildlife species, the degree of stochasticity might be lower (lower $\hat{\sigma}^{2}$ ).

Demographic fluctuations are due to finite population size and describe the variability of fecundity and survival rates of each individual independently. Goodman (1993) showed that this variable is only important for small population sizes because the individual variations cancel out if the population size is high. In the present study, it seems that the breed sizes, as well as the defined lower critical threshold $\left(n_{c}\right)$, are large enough to ignore this variable. Catastrophic events occur with a low probability but drastically reduce the population size within a short time period. For wildlife populations, catastrophes can include things like floods, wildfires, or epidemic plagues. These events could also occur for livestock breeds. Additionally, wars might be considered as catastrophic events for livestock breeds. For this reason, only observations from the years 1950 onwards (starting after World War II) were used, although this is in contrast with the demand of a large time span of observations (as mentioned above). In general, the modeling of catastrophic events is a problem in many PVA models (Ludwig, 1999).

Genetic effects describe reduced fitness of the individuals due to inbreeding. In practice, the rate of inbreeding is monitored by the breeding organizations, and tools for balancing genetic progress and inbreeding in small populations are available (Meuwissen, 1997; Sonesson and Meuwissen, 2001). Therefore, we assumed in this study that the inbreeding would not influence the population growth as long as the breed size remained above the defined critical value $n_{c}$ (bearing in mind that this is a simplification because the inbreeding rate is largely determined by the numbers 
of males in dairy cattle breeding schemes, which are not considered in the applied model).

As for all PVA methods based on time series, the errors of the observations of the population size should be as small as possible (e.g., Ludwig, 1999). The observations used in this study (number of milk-recorded females) are very reliable and satisfy this demand. Additionally, the costs for data collection are almost negligible. A drawback is that they represent only a proportion of the true number of females and, in general, this proportion did not remain constant over the time span of the observations. This fact that could be viewed as an argument against using milk-recording data, but the changes in this proportion were generally slow (e.g., for the Angler breed it was around 0.7 in 1970 and increased constantly to 0.85 in 2004; J. Piepenburg, LKV Schleswig-Holstein, personal communication, 2005). Furthermore, one can argue that milkrecorded females are much more important for a sustainable population size than are non milk-recorded females, because efficient dairy breeding schemes are based on milk-recording data and thus milk-recording females contribute much more to the future economical competitiveness of a breed. The use of milk-recorded females might, however, be problematic if extinction probability estimates of vastly different dual-purpose cattle breeds are to be compared, because they might differ substantially in the proportion of milk-recorded cows (the proportion may be high for a breed emphasizing dairy production and relatively low if beef is emphasized). For such a comparison, it would be better to have reliable data for the total number of femalesdata that are, however, much more difficult to collect.

Dennis et al. (1991) listed further assumptions of the PVA population growth diffusion approximation model. One important assumption is the approximate independence of the transitions of the Wiener drift process, i.e., the differences between 2 consecutive $X(t)$. As suggested by Dennis et al. (1991), this independence assumption was tested by analyzing the residuals of the linear regression applied to the cattle breed time series data sets by a Durbin-Watson test (Durbin and Watson, 1950). This test yields a Durbin-Watson statistic (it varies between 0 and 4 , and a value around 2 indicates no first-order autocorrelation among the residuals) together with a significance test $\left(\mathrm{H}_{0}=\right.$ no first-order autocorrelation, $\mathrm{H}_{1}=$ first-order autocorrelation among the residuals; this can be either positive or negative). The results show that this assumption was not violated in 5 of 6 cases (Table 2). A significant positive first-order autocorrelation was detected only for the German Gelbvieh, indicating that the results for this breed should be treated with caution. When the first-order autocorrelation is positive, $\sigma^{2}$ is under- estimated, and has to be increased by the level of autocorrelation. Positive autocorrelation is due to environmental factors that are correlated with the observations. Dennis and Otten (2000) suggested the inclusion of this correlated effect in the regression model. Unfortunately, in this study, no information was available about a putative correlated environmental effect. In such a case, perhaps a regression model that includes autocorrelated error terms would be appropriate. This possibility warrants further investigation. Negative autocorrelation can occur due to sampling error (Staples et al., 2004), but this was not detected in the present study. This fact underlines the high reliability of the observations used (number of milk-recorded cows).

A further assumption is that the population growth is density independent. In this study, we assumed that this assumption is approximately valid for a wide range of breed sizes, although this cannot be formally proven. Problems with this assumption might occur if a breed becomes either very large or very small. If all farmers in a certain region (e.g., within a country) switch to a particular breed, a population ceiling could be reached and the population growth rate of the breed in the region would be zero. The movement towards such an extreme situation might be accompanied by a decreasing, but still positive, population growth rate. However, the breeds considered in this study are far away from such an extreme situation. On the other hand, it is reasonable to assume that if a breed decreasing in numbers crosses a lower threshold of population size, the negative population growth rate might decrease (i.e., become more negative) very quickly and accelerate the extinction process. In this study, it was assumed that such a threshold is below the defined values of $n_{c}$.

The assessment of the precision of the PVA model is difficult because the pattern of an extinction of a livestock breed is more or less unknown. Hence, a simulation of this pattern to test the estimated population size or estimated extinction probability of the applied PVA model against the simulated parameters seems to be difficult. Therefore, the precision of the applied PVA model was tested during this study using a simplified approach. For each breed, the data was split into 2 groups; one-half of the data was used to estimate $\mu$ and $\sigma^{2}$. Based on these estimates, the CI of the last observed population size was predicted using [9] and [10], by using the log of the last true observed population size from the first half of the data set as the starting value $x_{q}$ in [9] and [10]. Finally, it was determined whether the true last observed population size $n_{q}$ was included in the obtained interval. This was the case for all 5 breeds (results not shown). The results appear 
to confirm that the model performed well, but it must be noted that this is only a very simple method for model validation. More research is needed in this field, perhaps by taking the ideas of McCarthy et al. (2001) into account. Additionally, one can reasonably assume that the recent trends in the change in population size provide more information about the future than do historical records. Therefore, it might be useful to test the effect of putting more weight on recent census data during the regression analysis.

\section{CONCLUSIONS}

Within livestock species, the prediction of the loss of genetic diversity within a given future time period due to putative extinction of breeds requires reasonable estimates of extinction probabilities (e.g., Simianer et al., 2003). Therefore, an attempt was made to estimate the extinction probability of 5 German cattle breeds using the population growth diffusion approximation PVA model of Dennis et al. (1991). This method is convenient to use and produced intuitively acceptable estimates. Furthermore, the assumptions of the model were determined to be at least approximately valid. Based on the results of the study the following recommendations can be given. First, extinction probabilities should be estimated not only for one future point in time $t_{e}$ but for every year up to an upper bound, which should not be too far in the future. This procedure would result in sets of extinction probabilities, one for every $t_{e}$. Second, the estimation of the expected loss of diversity should be conducted for every estimated set of extinction probabilities (i.e., for every $t_{e}$ ) in order to obtain an impression of the pattern of the loss with increasing $t_{e}$. Third, each extinction probability estimate should be reported with its level of confidence. One should also account for this level of confidence in the estimation of expected loss of genetic diversity, by repeatedly estimating loss in diversity after sampling the extinction probabilities from their respective CI. Finally, the extinction probabilities and the expected loss of diversity should be re-estimated whenever new observations on population size are available, to continually update and monitor the expected loss of diversity. With regard to this, the use of number of milk-recorded cows per year as observations is handy because it is renewed annually by the recording agency and thus would not demand extra costs for data collection.

\section{ACKNOWLEDGMENTS}

J. B. was supported by a grant from the German Academic Exchange Service (Deutscher Akademischer
Austauschdienst, DAAD). He thanks Ernst Kalm for excellent continuous support and for permission to stay at the Agriculture University of Norway in $\AA$ s to prepare this study.

\section{REFERENCES}

Beissinger, S. R., and M. I. Westphal. 1998. On the use of demographic models of population viability in endangered species management. J. Wildl. Manage. 62:821-841.

Bennewitz, J., and T. H. E. Meuwissen. 2005. A novel method for the estimation of the relative importance of breeds in order to conserve the total genetic variance. Genet. Sel. Evol. 37:315-337.

Burgman, M. A., S. Ferson, and H. R. Akcakaya. 1996. Risk assessment in conservation biology. Chapman and Hall, London, UK.

Dennis, B., P. L. Munholland, and J. M. Scott. 1991. Estimation of growth and extinction parameters for endangered species. Ecol. Monogr. 61:115-143.

Dennis, B., and M. R. M. Otten. 2000. Joint effects of density dependence and rainfall on abundance of San Joaquin Fox. J. Wildl. Manage. 64:388-400.

Durbin, J., and G. S. Watson. 1950. Testing for serial correlation in least square regression I. Biometrika 37:409-428.

Eding, J. H., R. P. M. A. Crooijmans, M. A. M. Groenen, and T. H. E. Meuwissen. 2002. Assessing the contribution of breeds to genetic diversity in conservation schemes. Genet. Sel. Evol. 34:613-633.

Eding, J. H., and T. H. E. Meuwissen. 2001. Marker based estimates of between and within population kinships for the conservation of genetic diversity. J. Anim. Breed. Genet. 118:141-159.

Ellner, S. P., J. Fieberg, D. Ludwig, and C. Wilcox. 2002. Precision of population viability analysis. Conserv. Biol. 16:258-261.

European Association for Animal Production (EAAP). 2005. EAAP animal genetic data bank, Hannover, Germany. Online. http:// www.tiho-hannover.de/einricht/zucht/eaap/.

FAO. 2000. World watch list for domestic animal diversity. 3rd ed. Rome, Italy. Online. http://www.fao.org/dad-is/.

Fieberg, J., and S. P. Ellner. 2000. When is it meaningful to estimate an extinction probability? Ecology 81:2040-2047.

Gandini, G. C., L. Ollivier, B. Danell, O. Distl, A. Georgoudis, E. Groeneveld, E. Martyniuk, J. A. M. van Arendonk, and J. A. Wolliams. 2004. Criteria to assess the degree of endangerment of livestock breeds in Europe. Livest. Prod. Sci. 91:173-182.

Ginzburg, L. R., L. B. Slobodkin, K. Johnson, and A. G. Bindman. 1982. Quasiextinction probabilities as a measure of impact on population growth. Risk Anal. 2:171-181.

Goodman, D. 1993. The demography of chance extinction. Pages 11-34 in Viable populations for conservation. M. E. Soule, ed. Cambridge University Press, Cambridge, UK.

Lande, R., and S. H. Orzack. 1988. Extinction dynamics of agestructured populations in a fluctuating environment. Proc. Natl. Acad. Sci. USA 85:7418-7421.

Ludwig, D. 1999. Is it meaningful to estimate a probability of extinction? Ecology 80:298-310.

McCarthy, M. A., H. P. Possingham, J. R. Day, and A. J. Tyre. 2001. Testing the accuracy of population viability analysis. Conserv. Biol. 15:1030-1038.

Meuwissen, T. H. E. 1997. Maximizing the response of selection with a predefined rate of inbreeding. J. Anim. Sci. 75:934-940.

Reed, J., L. S. Mills, J. B. Dunning, Jr., E. S. Menges, K. S. McKelvey, R. Frye, S. R. Beissinger, M.-Ch. Anstett, and P. Miller. 2002. Emerging issues in population viability analysis. Conserv. Biol. 16:7-19.

Reist-Marti, S. B., H. Simianer, J. Gibson, O. Hanotte, and J. E. O. Rege. 2003. Weitzman's approach and conservation of breed 
diversity: An application to African cattle breeds. Conserv. Biol. 17:1299-1311.

Shaffer, M. 1993. Minimum viable populations: Coping with uncertainty. Pages $69-86$ in Viable populations for conservation. M. E. Soule, ed. Cambridge University Press, Cambridge, UK.

Simianer, H., S. B. Marti, J. Gibson, O. Hanotte, and J. E. O. Rege. 2003. An approach to the optimal allocation of conservation funds to minimize loss of genetic diversity between livestock breeds. Ecol. Econ. 45:377-392.
Sonesson, A. K., and T. H. E. Meuwissen. 2001. Minimization of rate of inbreeding for small populations with overlapping generations. Genet. Res. 77:285-292.

Soule, M. E. (ed.). 1993. Viable populations for conservation. Cambridge University Press, Cambridge, UK

Staples, D. F., M. L. Taper, and B. Dennis. 2004. Estimating population trend and process variation for PVA in the presence of sampling error. Ecology 85:923-929.

Weitzman, M. L. 1993. What to preserve? An application of diversity theory to crane conservation. Q. J. Econ. 108:157-183. 